\title{
Structure-function scaling of bounded two-dimensional turbulence
}

\author{
W. Kramer, G. H. Keetels, H. J. H. Clercx, ${ }^{*}$ and G. J. F. van Heijst \\ Fluid Dynamics Laboratory, Department of Physics, Eindhoven University of Technology, P.O. Box 513, 5600 MB Eindhoven, \\ The Netherlands \\ (Received 15 March 2011; revised manuscript received 25 May 2011; published 10 August 2011)
}

\begin{abstract}
Statistical properties of forced two-dimensional turbulence generated in two different flow domains are investigated by numerical simulations. The considered geometries are the square domain and the periodic channel domain, both bounded by lateral no-slip sidewalls. The focus is on the direct enstrophy cascade range and how the statistical properties change in the presence of no-slip boundaries. The scaling exponents of the velocity and the vorticity structure functions are compared to the classical Kraichnan-Batchelor-Leith (KBL) theory, which assumes isotropy, homogeneity, and self-similarity for turbulence scales between the forcing and dissipation scale. Our investigation reveals that in the interior of the flow domain, turbulence can be considered statistically isotropic and locally homogeneous for the enstrophy cascade range, but it is weakly intermittent. However, the scaling of the vorticity structure function indicates a steeper slope for the energy spectrum than the KBL theory predicts. Near the walls the turbulence is strongly anisotropic at all flow scales.
\end{abstract}

DOI: 10.1103/PhysRevE.84.026310

PACS number(s): 47.27.ek, 47.10.ad, 47.27.De

\section{INTRODUCTION}

In his famous works on three-dimensional (3D) homogeneous isotropic turbulence Kolmogorov derived the inertial subrange scaling of the kinetic energy spectrum (see, for example, Frisch [1]). Applying the ideas proposed earlier by Richardson [2] he supposed that the redistribution of kinetic energy in the inertial subrange results in a net transport of kinetic energy from the integral scales of the flow (where energy injection takes place) toward the smallest (dissipative) scales. This process is known as the energy cascade of 3D turbulence. The arguments put forward by Kolmogorov are based on the assumption that the constant spectral energy flux is equal to the constant dissipation rate $\epsilon$ of kinetic energy. He also assumed that the kinetic energy $E(k)$ in this range depends on only $\epsilon$ and the wave number $k$. On dimensional grounds one then finds his famous five-thirds law: $E(k) \propto \epsilon^{2 / 3} k^{-5 / 3}$.

The physical picture of two-dimensional (2D) turbulence is different. The main significant difference between 2D and classical 3D turbulence consists of the formation and persistence of large coherent structures (vortices) in 2D turbulence. The emergence of large-scale coherent structures was already predicted on theoretical grounds by Onsager [3] and Fjørtoft [4]. With these predictions by Onsager and Fjørtoft at hand, attempts to formulate a phenomenological theory of 2D turbulence were put forward more than 40 years ago by Kraichnan [5,6], Batchelor [7], and Leith [8]. It is nowadays known as KBL theory. The statistical description of $2 \mathrm{D}$ turbulence in KBL theory is based on the assumptions of homogeneity and isotropy in the limit of infinite domain size. In the inviscid limit both the kinetic energy of the flow and the vorticity of fluid parcels are conserved. The latter actually implies an infinite number of conserved quantities, such as all area-averaged powers of the vorticity. Kraichnan [5] considered only two of the conserved quantities, the kinetic energy and the enstrophy (area-averaged square of

\footnotetext{
*Also at Department of Applied Mathematics, University of Twente, The Netherlands.
}

vorticity), and proposed the existence of a dual cascade in forced unbounded 2D turbulence. The dual cascade consists of the inverse energy cascade and the direct enstrophy cascade. Assuming a certain injection scale of kinetic energy (in wave-number space denoted by the wave number $k_{f}$ ) it is possible to make the following simplified picture of these cascades. By assuming the presence of a constant energy flux from the injection scale toward the largest scales accessible to the flow, and no enstrophy flux from the forcing scale to the smallest (and dissipative) scales, Kraichnan [5] predicted an inertial range scaling as $E(k) \propto \epsilon^{2 / 3} k^{-5 / 3}$, with $\epsilon$ the rate of cascade of kinetic energy per unit mass. The other limiting case concerns the situation with the presence of a constant enstrophy flux from the injection scale toward the dissipative scales, and no energy flux to the largest scales of motion. For this case an inertial range according to $E(k) \propto \chi^{2 / 3} k^{-3}$ has been predicted. Here $\chi$ is the enstrophy dissipation rate.

The first direct numerical simulations (DNS) of forced 2D turbulence with periodic boundary conditions was carried out by Lilly [9] in order to confirm the existence of the dual cascade. These numerical studies were restricted to a resolution of $64^{2}$ grid points. Although this definitely represents too low of a resolution, these results indicated for the first time the presence of a $k^{-5 / 3}$ and a $k^{-3}$ inertial range. To obtain the dual cascade, the domain size, forcing scale, and dissipative scales should be sufficiently separated from each other, and small-banded forcing in wave number space is required. Obviously, numerical experiments aimed at simultaneously generating both one-to-two decade inverse energy and direct enstrophy cascades require therefore extremely high resolutions. Only very recently Boffetta [10] reported on a very-high-resolution (up to $16384^{2}$ grid points) simulation of stationary $2 \mathrm{D}$ turbulence. He used a forcing that is narrow banded in space and short correlated in time with a forcing scale $l_{f}=L / 100$, with $L$ the box size and $l_{f} \propto 1 / k_{f}$. Thus approximately two decades are available for both the inverse energy cascade (for $k<k_{f}$ ) and the direct enstrophy cascade (for $k>k_{f}$ ). This DNS of the 2D Navier-Stokes equations indeed reproduces, for the first time, the dual cascade scenario predicted by Kraichnan. 
In this paper we consider the statistical properties of forced 2D turbulence in bounded domains with no-slip boundaries. In numerical studies on forced 2D turbulence in a periodic box (for an overview, see Ref. [11]), it is common practice to use additional friction mechanisms in order to achieve a statistically steady state. In particular, artificial friction at large scales is generally applied to circumvent the pile-up of energy (due to the inverse energy cascade) at domain-sized scales. As observed in the high-resolution simulations of Boffetta [10] the presence of friction has dramatic consequences for the statistical properties of the smallest scales of motion. In the present simulations the no-slip boundary provides a natural sink for the kinetic energy that can balance the injection of energy by the external forcing such that a steady state is achieved. In this way the use of a volumetric drag force can completely be avoided. Therefore, it is challenging to determine the statistical properties of the small scales of turbulence in the interior of the flow domain with no-slip boundaries, as well as close to the sidewalls.

Another remarkable observation for forced 2D turbulence on a periodic domain by Smith and Yakhot [12,13], already predicted by Kraichnan [5], is the formation of a large-scale vortical structure, called a condensate. Here all the energy that cascades from the injection scale toward the large scales $(k \approx$ $1)$ is collected in this condensate. The emerging large-scale structure turned out to have a dipole-like character. Indications of this phenomenon from numerical studies had already been reported by Hossain et al. [14]. It is anticipated that the no-slip boundaries are able to prevent energy accumulation on the largest scales of motion and the subsequent formation of a condensate (due to the inverse energy cascade). Smith and Yakhot $[12,13]$ found that in a condensation regime the selfsimilarity of the small-scale statistics is lost. It is examined in this paper whether the no-slip boundaries sufficiently prevent the accumulation of energy in domain-sized coherent vortices and subsequently sustain the self-similarity at the small scales.

A difficulty that may keep the small-scale structure from obtaining KBL scaling is the injection of high-amplitude vorticity filaments, generated at the no-slip domain boundaries, into the bulk of the flow. Note that this process may affect the isotropy and local homogeneity of the turbulence. Furthermore, Clercx and van Heijst [15] provide evidence that in decaying 2D turbulence boundary-layer detachment can result in an inverse energy cascade in the wall region. Wells et al. [16] used a forcing mechanism aimed solely at generating turbulence by vorticity production at no-slip sidewalls (without direct vorticity production in the interior). They achieved this by oscillating the container with a certain frequency, thus generating Stokes boundary layers. This process indeed results in the production of small-scale vorticity at the domain boundaries that accumulate in thin boundary layers. These boundary layers eventually detach and move subsequently into the interior of the flow domain. The energy spectra obtained by Wells and coworkers [16] indeed suggest that these vorticity layers feed both an inverse energy cascade and a direct enstrophy cascade. In the present study the container remains fixed, and we apply an external forcing mechanism (with vorticity production in the interior of the domain). An important question is whether the interaction of the turbulence with the no-slip boundaries and subsequent detachment of boundary layers, thus transporting vorticity into the interior, has an important effect on the enstrophy transfer rate of the internal flow.

In this paper we take a dual approach for determining statistical properties of forced $2 \mathrm{D}$ turbulence in a domain bounded by no-slip walls. A square bounded domain is used to investigate how the statistics in the interior changes. For determining statistical properties near the domain boundaries simulations in a rectangular periodic channel domain with two (opposite) no-slip walls are used. In the following sections the problem and the numerical models used are described. Then we give a short description on the flow structures that appear. For both problems, the isotropy and self-similarity properties of turbulence are determined. Finally, the power-law scaling in the enstrophy-cascade range is investigated.

\section{PROBLEM DESCRIPTION}

Consider an incompressible fluid of unit density, $\rho=1$, in a domain $\Omega \in \mathbb{R}^{2}$, which evolves according to the Navier-Stokes equations

$$
\partial_{t} \mathbf{u}+(\mathbf{u} \cdot \nabla) \mathbf{u}+\nabla p-v \Delta \mathbf{u}=\mathbf{f} \quad \text { in } \quad \Omega \times[0, T]
$$

and the continuity condition

$$
\nabla \cdot \mathbf{u}=0 \quad \text { in } \Omega \times[0, T],
$$

where $\mathbf{u}=[u(\mathbf{x}, t), v(\mathbf{x}, t)]$ is the Eulerian velocity, $\mathbf{x}=(x, y)$ denotes the position vector in a Cartesian coordinate frame, $p=p(\mathbf{x}, t)$ is the scalar kinetic pressure, $v$ the kinematic viscosity, and $\mathbf{f}=\mathbf{f}(\mathbf{x}, t)$ denotes the amount of external force per unit area. For 2D incompressible flows it is common to introduce the stream function $\psi$, and the relation of $\psi$ with the velocity field is $u=\partial_{y} \psi$ and $v=-\partial_{x} \psi$. The complexity of the flow is controlled by the Reynolds number $\operatorname{Re}=U L / v$, where $U$ represents a typical velocity and $L$ a typical length scale. The Reynolds number is a measure of the relative strength of the advective versus the viscous term. In the present study the flow domain is (partly) bounded by a nonmoving impermeable wall, which implies that the velocity component normal to the wall equals zero. Furthermore, the (viscous) no-slip condition implies that the tangential velocity component is zero at the wall. The combination of these boundary conditions gives

$$
\mathbf{u}(\mathbf{x}, t)=0 \quad \mathbf{x} \in \partial \Omega, \quad t \in[0, T],
$$

which is essentially a Dirichlet boundary condition for $\mathbf{u}$. Note that Eq. (1) contains second-order derivatives, so two boundary conditions are required for the existence of a unique solution.

On a rectangular bounded geometry

$$
\Omega=\left\{\mathbf{x} \in \mathbb{R}^{2} \mid-L \leqslant x<L,-W \leqslant y \leqslant W\right\},
$$

it is also possible to define periodic boundary conditions, which means that the value of the solution at the boundary $x=-L$ equals the value at the boundary $x=L$. The same periodicity may hold for the boundaries at $y=W$ and $y=-W$ (but for the periodic channel geometry these walls are of the no-slip type). The formulation is completed by appending the initial condition

$$
\mathbf{u}(\mathbf{x}, 0)=0, \quad \mathbf{x} \in \Omega .
$$


For convenience the Navier-Stokes equations in velocitypressure or primitive variables (1) can be rewritten in the velocity-vorticity formulation by taking the curl of Eq. (1) and applying some vector identities to arrive at

$$
\partial_{t} \omega+(\mathbf{u} \cdot \nabla) \omega-v \Delta \omega=q \quad \text { in } \quad \Omega \times[0, T],
$$

where

$$
\omega=(\nabla \times \mathbf{u}) \cdot \mathbf{e}_{z}=\partial_{x} v-\partial_{y} u
$$

is the scalar vorticity and $q=(\nabla \times \mathbf{f}) \cdot \mathbf{e}_{z}$ is the $z$ component of the curl of the external forcing. Note that the velocityvorticity formulation is scalar valued in two dimensions. The boundary conditions on $\partial \Omega$ for the velocity-vorticity formulation are defined in terms of the velocity since no physical boundary condition is a priori available in terms of the vorticity.

The forcing in terms of $q$ in the vorticity equation (5) is defined as a Markov chain process

$$
q\left(t_{n}, \mathbf{x}\right)=c_{r} q\left(t_{n-1}, \mathbf{x}\right)+\left|A_{p}\right|\left(1-c_{r}^{2}\right)^{1 / 2} w(\mathbf{x}),
$$

where

$$
c_{r}=\frac{1-\delta t / \tau_{c}}{1+\delta t / \tau_{c}}
$$

is the correlation factor with correlation time $\tau_{c}$ and $\left|A_{p}\right|$ the amplitude, which is static in time (for details, see Ref. [17]). The function $w(\mathbf{x})$ takes completely random values at each time step $\delta t$. The spatial structure of $w(\mathbf{x})$ is generated by considering the Fourier transform

$$
w(\mathbf{x})=\sum_{k_{1} \leqslant|\mathbf{k}| \leqslant k_{2}} \exp \left(i \theta_{\mathbf{k}}\right) \exp (i \mathbf{k} \cdot \mathbf{x}) .
$$

The phase $\theta_{\mathbf{k}} \in[0,2 \pi]$ is drawn at each time step $\delta t$ from a uniform distribution that is independent for each wave vector in the shell $k_{1} \leqslant|\mathbf{k}| \leqslant k_{2}$. This forcing protocol, originally introduced by Lilly [9], is commonly used in numerical simulation of $2 \mathrm{D}$ turbulence.

\section{NUMERICAL SETUP}

Two spectral methods are applied to solve the vorticity equation (5) in bounded domains. One method, based on the expansion of the flow variables in Fourier and Chebyshev polynomials, is used to solve the flow in a periodic channel. The other method consists of a standard Fourier method, but the solid boundaries are implemented using a volume penalization technique. Here we will give a short description of both methods; more details can be found in Refs. [18,19].

In the case of the periodic channel the equations are solved numerically using a Fourier-Chebyshev pseudospectral method in the domain $\Omega$. We use the formulation proposed by Daube, where the velocity-vorticity equation (5) is supplemented by two Poisson equations for the velocity components [20]. The domain $\Omega$ is spanned by the rectangle $[-4,4) \times[-1,1]$ (in this particular study we limit the domain to an aspect ratio $\frac{L}{W}=4$ with the half-width fixed at $W=1$ and the half-length of the channel at $L=4$ ). The no-slip walls of the channel are located at $y= \pm 1$. The velocity and vorticity are expanded in a truncated series of Fourier polynomials for the $x$ direction and in a truncated series of Chebyshev polynomials for the nonperiodic $y$ direction. For the numerical computation of the vorticity evolution, a semi-implicit time discretization scheme is applied to the vorticity equation (5). The linear terms in (5) are discretized by the backward differencing formula (BDF) of third order. The nonlinear term is extrapolated from the three previous time levels; for details, see Refs. [18,21]. Applying the spatial and time discretizations yields a system of equations for the spectral coefficients for the velocity and vorticity, which can be resolved using a specially tailored Gaussian elimination technique. The vorticity values at the walls are not known a priori. Enforcing the vorticity definition (6) with an influence matrix technique yields the correct boundary values for the vorticity at the walls [20,22].

In the volume-penalization approach fluid-wall interaction is no longer described by demanding no-slip boundary conditions. The basic idea is to embed the square flow domain, $[-1,1] \times[-1,1]$, in a larger domain with porous walls. The porous walls are modeled by adding a Darcy drag term to the Navier-Stokes equations, which yields the penalized vorticity equation,

$$
\frac{\partial \omega}{\partial t}+(\mathbf{u} \cdot \nabla) \omega-v \Delta \omega=\frac{1}{\epsilon}(\nabla \times H \mathbf{u}) \cdot \mathbf{e}_{z}+q .
$$

Here $\epsilon$ is the penalization parameter and $H$ a mask function, which has values 1 inside the porous walls and 0 for the flow domain. An advantage to the penalization approach is that periodic boundary conditions can be used for the enlarged domain. This permits to use a standard dealiased Fourier spectral method for solving the penalized NavierStokes equations. A third-order BDF scheme with exact time differentiation of the diffusion term is applied [19]. Carbou and Fabrie [23] have shown that the $\mathrm{L}_{2}$ norm of the differences in the velocity $\mathbf{u}$ and velocity gradients $\nabla \mathbf{u}$ of the penalized Navier-Stokes equations and the Navier-Stokes equations with no-slip boundary conditions is proportional to $\sqrt{\epsilon}$. Inside the porous wall an asymptotically thin boundary layer exists with thickness proportional to $\sqrt{v \epsilon}$. The Gibbs oscillations that derive from this thin boundary layer are not influencing the solution and can be removed by a mollification technique [19]. For the mollified solution spectral accuracy is restored for the fluid domain except for a thin layer at the boundary.

The Fourier spectral method with volume penalization has recently been successfully applied to several decaying 2D turbulence studies (including Lagrangian dispersion studies in such flows) and 2D plasma turbulence on domains bounded by sidewalls. In particular for square [24,25], circular [25-29], and elliptical domains $[25,30]$.

In Table I the settings used in the numerical simulations are specified. Normal to the wall about 10 grid points are used to resolve the viscous boundary layer. This determines the number of active modes for simulations on the square domain, where a uniform grid is used. The distribution of grid points normal to the wall in the periodic channel domain is finer near the wall than in the interior (as Chebyshev collocation points condense near the boundaries). Hence, we can use a smaller number of Chebyshev modes in the periodic channel than the number of Fourier modes in the square bounded domain. The grid spacing in the periodic direction is about equal to the interior grid spacing in the wall-normal direction. The time step is limited by CFL conditions and is small enough to resolve the 
TABLE I. Numerical parameters for the simulations on a square domain and periodic channel domain with aspect ratio $\frac{L}{W}=4$. Here, $v$ is the kinematic viscosity, $N$ and $M$ are the number of grid points in the $x$ and $y$ (wall-normal for the periodic channel) direction, respectively, $\Delta t$ the time step, $A_{p}$ the forcing amplitude, $\tau_{c}$ the forcing correlation time, $k_{f}$ the forcing wave number, and $l_{f}$ the typical forcing length scale.

\begin{tabular}{lcccccccc}
\hline \hline Domain & $v$ & $N$ & $M$ & $\Delta t$ & $A_{p}$ & $\tau_{c}$ & $k_{f}$ & $l_{f}$ \\
\hline Square & $10^{-4}$ & 1364 & 1364 & $4 \times 10^{-5}$ & 6 & $10^{-2}$ & {$[7 \pi, 9 \pi]$} & 0.25 \\
Channel & $10^{-4}$ & 2048 & 512 & $4 \times 10^{-5}$ & 8 & $10^{-2}$ & {$[7 \pi, 9 \pi]$} & 0.25 \\
\hline \hline
\end{tabular}

correlation time of the forcing. The viscosity and the forcing amplitude are chosen in such a way that the integral-scale Reynolds number of both simulations is about 20000 . The forcing is limited to the wave numbers $7 \pi \leqslant\left|k_{f}\right| \leqslant 9 \pi$, which relates to a forcing length scale of about one-eighth of the domain width. The forcing length scale must be large to obtain a long direct enstrophy cascade range, but has to be small enough to produce an isotropic forcing field. For the bounded square domain we ensure that Fourier modes with $k_{x}=0$ or $k_{y}=0$ are not present in the forcing, such that the forcing does not exert a net torque on the fluid.

\section{STATISTICALLY STEADY STATE}

In Fig. 1 the evolution of the total kinetic energy $E=$ $\frac{1}{2} \int_{\Omega}|\mathbf{u}|^{2} d x d y$ and enstrophy $Z=\frac{1}{2} \int_{\Omega} \omega^{2} d x d y$ are given for the simulation in the square domain. It can be deduced that the flow slowly converges toward a statistically steady state. The maximum value of the (time-dependent) Reynolds number $\operatorname{Re}(t)$ is approximately 25000 . A similar observation
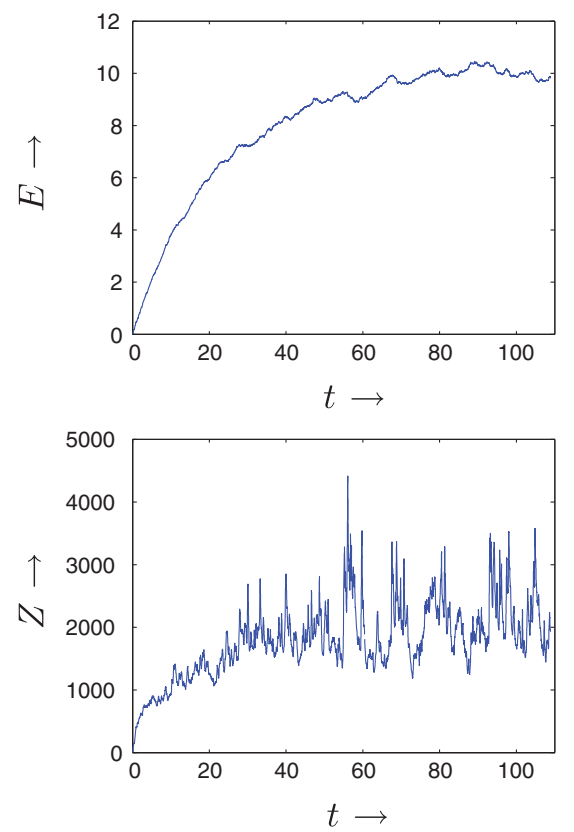

FIG. 1. (Color online) Total kinetic energy (top) and enstrophy (bottom) vs dimensionless time (where $t=1$ corresponds to a typical eddy turnover time of the larger vortices) of a forced turbulence simulation in a square bounded domain. The integral-scale Reynolds number is approximately 25000 . can be made for the periodic channel domain, where the Reynolds number reaches $\operatorname{Re} \approx 21000$. Note that in the case of periodic boundary conditions (in both coordinate directions) the applied forcing mechanism would result in an unbounded growth of the total kinetic energy. In the absence of bottom friction the energy input by the forcing has to be balanced by viscous dissipation, thus $\langle(\mathbf{f}, \mathbf{u})\rangle=2 v\langle Z\rangle$ with $(\mathbf{f}, \mathbf{u})=$ $\int_{\Omega} \mathbf{f}(\mathbf{x}, t) \cdot \mathbf{u}(\mathbf{x}, t) d x d y$ and $\langle\cdot \cdot\rangle$ denoting an ensemble average. Usually it is assumed that it is legitimate to adopt the dynamical system concept of ergodicity such that ensemble averages can be replaced by time averages, or $\lim _{T \rightarrow \infty} \frac{1}{T} \int_{0}^{T} f(\mathbf{u}) d t=$ $\langle f(\mathbf{u})\rangle$. The production of vorticity at the domain boundaries results in a sufficiently high dissipation rate of kinetic energy that is able to balance the energy injection of the external forcing. The peaks in the enstrophy signal shown in the bottom panel of Fig. 1 are mainly a result of intensive flow-wall interactions. To illustrate this we first introduce the enstrophy balance,

$$
\frac{d Z}{d t}=v \oint_{\partial \Omega} \omega(\mathbf{n} \cdot \nabla) \omega d s-2 v P+(\omega, q),
$$

with $d s$ an infinitesimal element tangential to the boundary, $P=\frac{1}{2} \int_{\Omega}|\nabla \omega|^{2} d x d y$ the palinstrophy of the flow, and $(\omega, q)=\int_{\Omega} \omega(\mathbf{x}, t) q(\mathbf{x}, t) d x d y$. It is verified that the first two terms in the enstrophy balance (11) are two orders of magnitude larger than the last term which quantifies the vorticity injection of the external forcing. This indicates that the steady state in the total kinetic energy is indeed achieved by production of vorticity at the domain boundaries.

In Fig. 2 snapshots of the vorticity field and the corresponding stream function are given for two different times. Several vortices can be recognized with different sizes and strengths. The larger vortices are a direct result of the forcing, whereas the smallest vortices originate from the thin detached boundary layers [31]. In the present simulations the large-scale flow patterns can be associated with the strongest vortices in Fig. 2 . Since the angular momentum $L$ with respect to the origin $(0,0)$ can be written as $L=2 \int \psi d x d y$, it is obvious from the circulation of the dominant large-scale coherent structure (and thus the sign of $\psi$ ) that the angular momentum changes sign between $t=90$ and $t=110$. The energy does not pile up in the dominant vortices due to a strong dissipation of energy during the interaction with the no-slip sidewalls. The formation of a condensate, as observed in the simulations of forced 2D turbulence on a periodic domain without bottom friction by Smith and Yakhot [12,13], is absent. In these simulations almost all the vorticity is eventually contained in the two cores of a single dipolar vortex (one patch with negative and another with positive vorticity). The no-slip sidewall in our simulations clearly prevents the accumulation of energy in domain-sized coherent structures and hence prevents the formation of a condensate.

A similar observation can be made for the rectangular periodic channel domain. As shown by the stream function in Fig. 3 the flow consists of an array of four circulations cells. Each cell corresponds with one strong large-scale vortex or a cluster of vortices. The dimension of each circulation cell is limited by the domain width. Hence, one can expect that the number of circulation cells matches approximately the aspect 

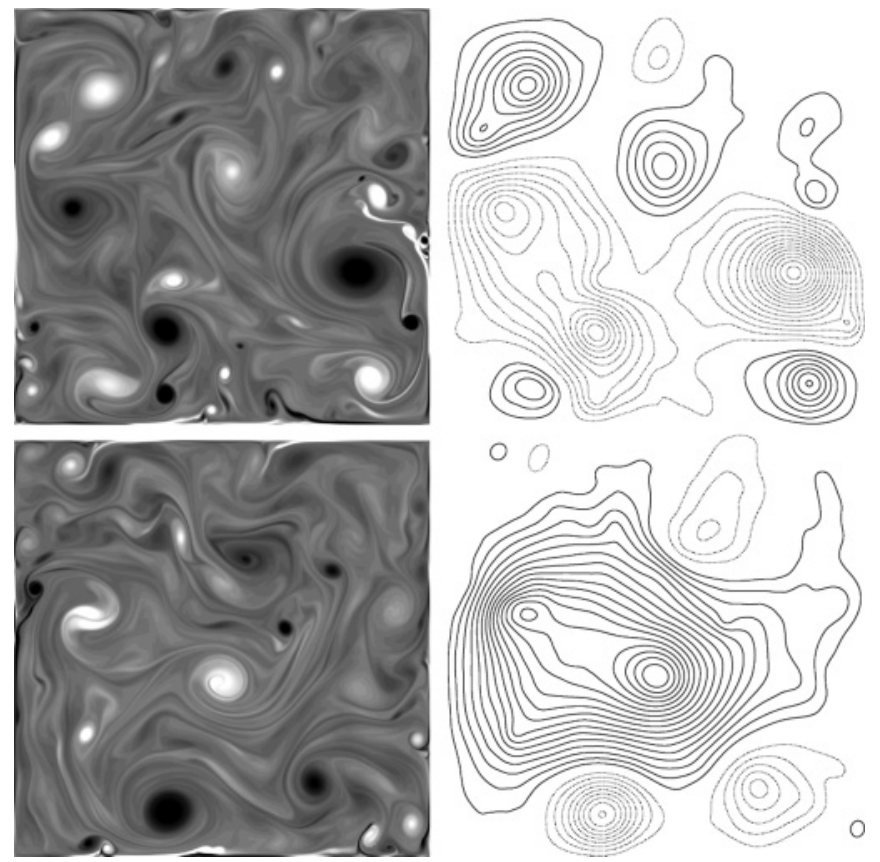

FIG. 2. Snapshots of the vorticity distribution and the stream function of forced 2D turbulence in the square domain taken at $t=90$ (top row) and $t=110$ (bottom row). The vorticity ranges from $\omega=-120$ to $\omega=+120$ with 30 gray levels. The stream function is shown with an interval of 0.1 . The solid isolines correspond with positive values of $\psi$, and the dashed isolines represent negative values of $\psi$.

ratio of the domain. Again, the smallest vortices derive from vorticity boundary layers that are detached from the wall [17].

\section{ISOTROPY}

The spatial structure of the flow can be analyzed by considering the statistical properties of velocity differences $\delta \mathbf{u}(\mathbf{x}, \mathbf{r}, t)=\mathbf{u}(\mathbf{x}+\mathbf{r}, t)-\mathbf{u}(\mathbf{x}, t)$. For statistically stationary flows it is allowed to drop the time dependence; we are thus left with $\delta \mathbf{u}(\mathbf{x}, \mathbf{r}, t)=\delta \mathbf{u}(\mathbf{x}, \mathbf{r})$. The velocity difference can be decomposed in components parallel and perpendicular
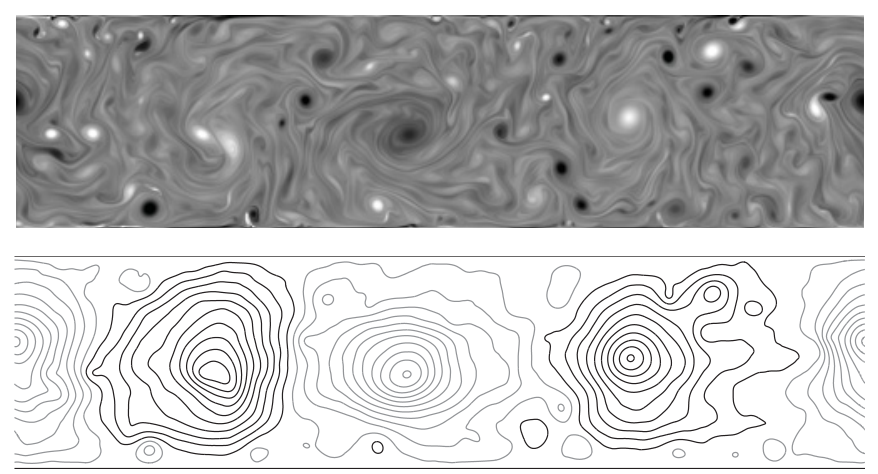

FIG. 3. Snapshots of the vorticity and the stream function in the periodic channel domain. The vorticity ranges from $\omega=-120$ to $\omega=+120$ with 30 gray levels. The stream function is shown with an interval of 0.1 . The black isolines correspond with positive values of $\psi$, and the gray isolines represent negative values of $\psi$. to $\mathbf{r}: \delta u_{\|}(\mathbf{x}, \mathbf{r})=\delta \mathbf{u}(\mathbf{x}, \mathbf{r}) \cdot \mathbf{r} / r$ and $\delta u_{\perp}(\mathbf{x}, \mathbf{r})=\delta \mathbf{u}(\mathbf{x}, \mathbf{r}) \cdot \mathbf{r}_{\perp} / r$, respectively, with $r=|\mathbf{r}|$ and $\mathbf{r}_{\perp}=\hat{\mathbf{k}} \times \mathbf{r}$ (with $\hat{\mathbf{k}}=(0,0,1)$ the unit vector perpendicular to the plane of the flow). By assuming homogeneity and isotropy we can unambiguously drop the $\mathbf{x}$ dependence and replace $\mathbf{r}$ by $r$ in statistical averages. The structure functions of different orders $p$ for the longitudinal and transverse velocity components are $S_{p}^{\|}(r)=$ $\left\langle\left|\delta u_{\|}\right|^{p}\right\rangle$ and $S_{p}^{\perp}(r)=\left\langle\left|\delta u_{\perp}\right|^{p}\right\rangle$, respectively. The scaling of the structure function $S_{p}$ is given by

$$
S_{p}(r) \sim r^{\zeta_{p}}
$$

with $\zeta_{p}=h p$ the scaling exponent [assuming that $\delta u(r) \sim r^{h}$ ]. Note that the structure functions are much better suited for application in bounded domains than the spectra, as computation of spectra on a nonperiodic domain requires a spatial filtering of the solution near the wall.

The relation between the transverse second-order structure function $S_{2}^{\perp}(r)$ and the longitudinal second-order structure function $S_{2}^{\| l}(r)$,

$$
S_{2}^{\perp}(r)=\left(1+\frac{r}{2} \frac{d}{d r}\right) S_{2}^{\|}(r),
$$

permits to verify whether the small-scale statistics is consistent with the crucial KBL assumption that the flow is locally homogeneous and isotropic. The procedure is straightforward: First, one computes both structure functions, and then one computes an estimate for $S_{2}^{\perp}(r)$ by using $S_{2}^{\|}(r)$ and relationship (13). Strong deviations between the estimate for $S_{2}^{\perp}(r)$ and the direct determination of $S_{2}^{\perp}(r)$ indicate the presence of anisotropy or local inhomogeneity in the flow.

Figure 4 shows the transverse second-order structure function of the velocity obtained from a forced 2D turbulence simulation in a square box (with width 2). The second-order structure function is either directly evaluated in a square box in the center of the domain or indirectly by applying Eq. (13). Various box sizes with a width ranging from 0.6 to 1.2 have

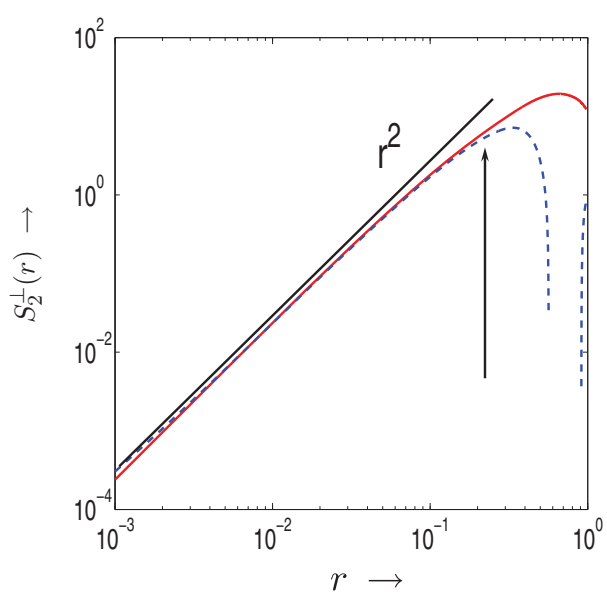

FIG. 4. (Color online) Transverse second-order structure function of the velocity, $S_{2}^{\perp}(r)$ (solid line). The arrow denotes the length scale of the forcing. The dashed line corresponds with the isotropic estimate for the transverse second-order structure function based on the longitudinal structure function. The structure functions are computed in a square box with a width 1.0 in the center of the flow domain (the square computational domain has a width 2). 
been considered, and no significant differences have been observed for the statistical quantities in the present study. Therefore, all the presented results for the square domain are calculated in a box centered in the interior with a width equal to one. Figure 4 also shows a comparison of the transverse second-order structure function, numerically evaluated from the available flow field, with the computed estimate obtained with Eq. (13), thus based on the longitudinal second-order structure function. The result is in good agreement for scales smaller than the forcing scale, which supports the assumption that the flow can be considered as statistically isotropic and locally homogeneous. The structure function $S_{2}^{\perp}(r)$ is proportional with $r^{2}$ for the smallest separations and flattens when moving to the forcing length scale.

While isotropy for scales smaller than the forcing scale is observed in the interior, this is not likely near the walls. In viscous boundary layers the wall-normal velocity is much smaller than the tangential velocity. Figure 5 displays the transverse velocity structure function calculated along four different lines parallel to the no-slip sidewalls in the periodic channel. Along the center line of the channel $(y=0)$ isotropy is again retrieved for all scales smaller than the forcing scale, and $S_{2}^{\perp} \propto r^{2}$. Moving toward the wall the statistical properties of the turbulent flow become more anisotropic, in particular for $y=0.95$ and $y=0.99$. The line $y=0.99$ is located inside the boundary layer based on the $99 \%$ velocity criterion, but well outside the viscous sublayer. While the anisotropy is strongest for the largest scales, isotropy is even lost for the scales in the viscous subrange; see Fig. 5.

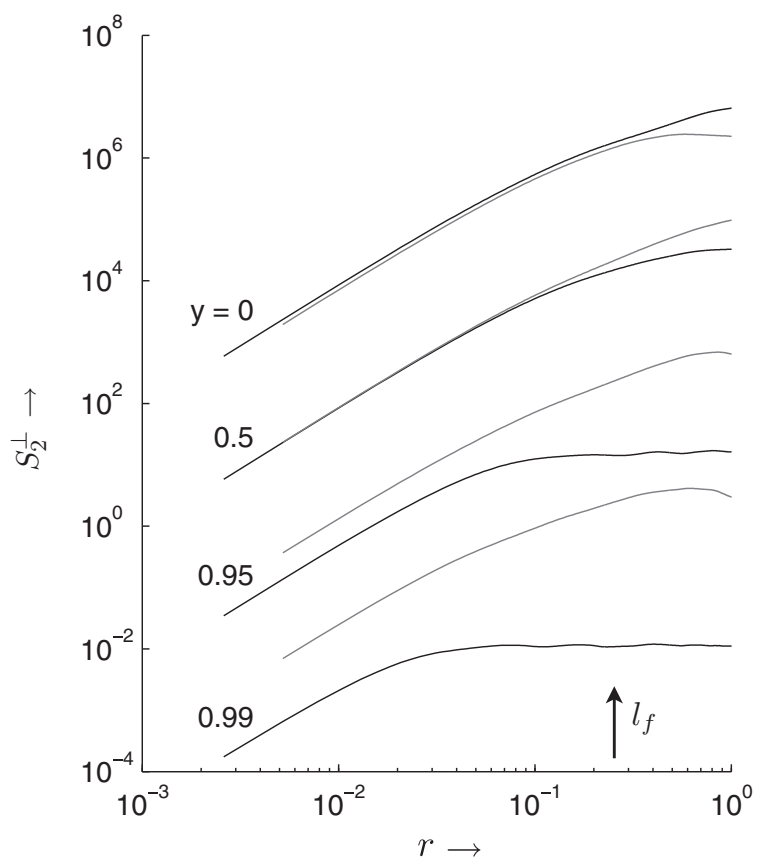

FIG. 5. Transverse second-order structure function of the velocity computed over the lines $y=0.99, y=0.95, y=0.5$, and $y=0$ (ranging from the sidewall to the center of the channel, respectively). Each subsequent plot is shifted by a factor $10^{2}$. The gray line in each panel corresponds with the isotropic estimate for the transverse second-order structure function $S_{2}^{\perp}(r)$, based on the longitudinal structure function; see Eq. (13).
A special note needs to be made about the scaling exponent $\zeta_{p}$ of the structure functions. The power-law scaling exponent of a structure function $S_{p}(r)$ is bounded by its order, $\zeta_{p} \leqslant p$ (see, for example, Ref. [32]). Therefore, the comparison with the exponent of the energy spectrum has some limitations. The classical $k^{-5 / 3}$ scaling of the energy spectrum in the inverse energy cascade relates to a $r^{2 / 3}$ scaling of the second-order structure function of the velocity. Evaluating the scaling in the enstrophy-cascade range $\left[E(k) \propto k^{-3}\right]$ is not straightforward as the corresponding $r^{2}$ scaling for the second-order velocity structure function, shown in Fig. 4, is equal to the upper bound. Hence, steeper energy spectra, for instance, in the viscous subrange, always result in $S_{2}^{\perp}(r) \propto r^{2}$ scaling. In Sec. VII we investigate the second-order structure function of the vorticity, $S_{2, \omega}$, for extracting the scaling of the energy spectrum, as it has a slope usually well separated from this upper bound.

\section{INTERMITTENCY AND SELF-SIMILARITY}

A straightforward method to analyze the scale dependence of the statistical properties of turbulence is to investigate the probability density function (pdf) of the vorticity increments $\delta \omega(\mathbf{r})=\omega(\mathbf{x}+\mathbf{r})-\omega(\mathbf{r})$ at different separations $r=|\mathbf{r}|$. Figure 6 presents the pdf of the vorticity increments for seven separations in the range $r \lesssim l_{f}$. It is observed that the pdf has a similar shape for all separations. A Gaussian core can be recognized with exponential tails. The similarity in the shape of the pdf suggests scale-invariant statistics.

This particular shape of the pdf is typically observed in two-dimensional turbulence. The general picture is that the heavy exponential tails are related to the presence of coherent vortices in the flow. The same pdf is observed in the interior of the periodic channel (see data obtained for $y=0$, shown in Fig. 7). Near the wall a totally different pdf is observed, $P(s) \sim \exp \left(-c|s|^{-1 / 2}\right)$, where $s=\delta \omega$ and $c$ a constant. The probability of observing very weak and very strong vorticity increments is increased at the expense of fluctuations at average intensity. Boffetta [33] observed a similar pdf in numerical simulations of $2 \mathrm{D}$ turbulence with Ekman friction. He argued that the visual counterpart is the

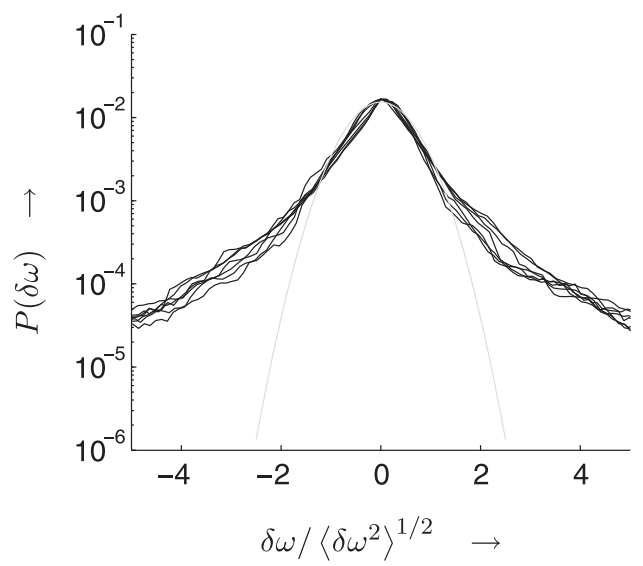

FIG. 6. Probability density function $P(\delta \omega)$ of the vorticity increments $\delta \omega$ for separations $r \lesssim l_{f}(r=1 / 256,1 / 128,1 / 64,1 / 32$, $1 / 16,1 / 8$, and $1 / 4$ ) in the interior of the square bounded domain. A Gaussian distribution is given in gray for comparison. 


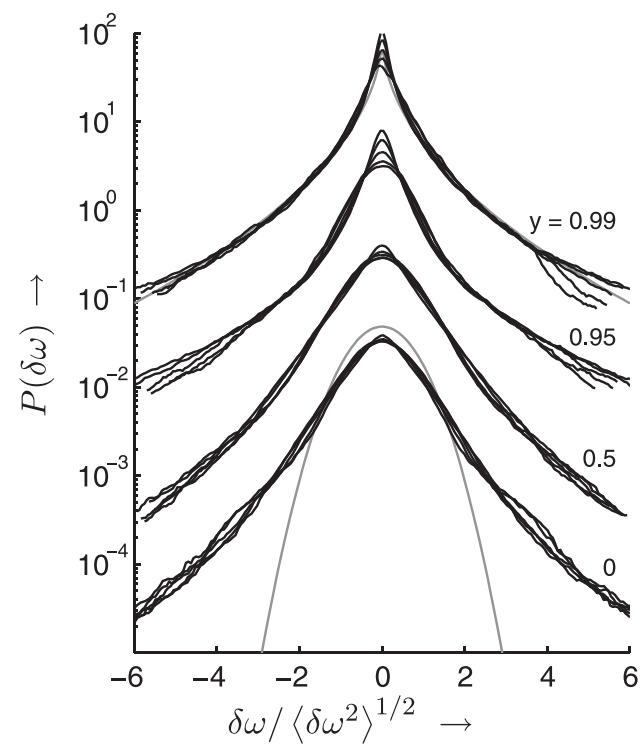

FIG. 7. Probability density function $P(\delta \omega)$ of the vorticity increments for separations $r \lesssim l_{f}(r=1 / 64,1 / 32,1 / 16,1 / 8$, and $1 / 4)$ in the periodic channel domain for the lines $y=0, y=0.5, y=0.95$, and $y=0.99$ with each subsequent plot shifted upward for clarity. The gray line in the lower plot depicts a Gaussian distribution with the same variance as in Fig. 6, while in the upper plot the gray line gives the distribution $P(s) \sim \exp \left(-c|s|^{-1 / 2}\right.$ ) (which are mostly buried in the different pdfs).

organization of the vorticity field into "quiescent" areas (where the vorticity changes smoothly) and active regions (dominated by filaments). The vorticity in the boundary layer is a response to the internal flow structures. The large circulation cells present in the interior flow yield a relatively smooth vorticity at the wall. However, when the boundary layers detach from the wall, high-amplitude vorticity filaments occur perpendicular to the wall.

The self-similar scaling of the form (12) is motivated by the scale invariance of the Euler equations. It is assumed that Navier-Stokes flow in the limit of $v \rightarrow 0$ will obtain self-similar scaling. However, as the forcing scale and the dissipation scale are only weakly separated in our simulations, the presence of viscous dissipation can be expected to be relevant for all separations smaller than the forcing scale. Since the Stokes equation is not scale invariant, it can be expected that self-similarity will no longer hold.

A more accurate method for the detection of intermittency effects is proposed by Benzi et al. [34]. They showed that locally homogeneous, isotropic turbulence obeys a more general scaling relation of the form

$$
S_{p}(r) \sim S_{3}^{\tilde{\zeta}_{p}}
$$

The scaling (14) extends the inertial range property (12), which is strictly valid for $r_{d} \lesssim r \lesssim l_{f}$. The relative scaling exponent $\tilde{\zeta}_{p}$ allows a more accurate determination and hence a more reliable detection of the presence of either extended self-similarity (ESS) or intermittency.

The relative scaling exponent can now be expressed as $\tilde{\zeta}_{p}=p / 3+\gamma_{p}$, where $\gamma_{p}$ is a measure of the degree of intermittency. Babiano et al. [35] determined the relative
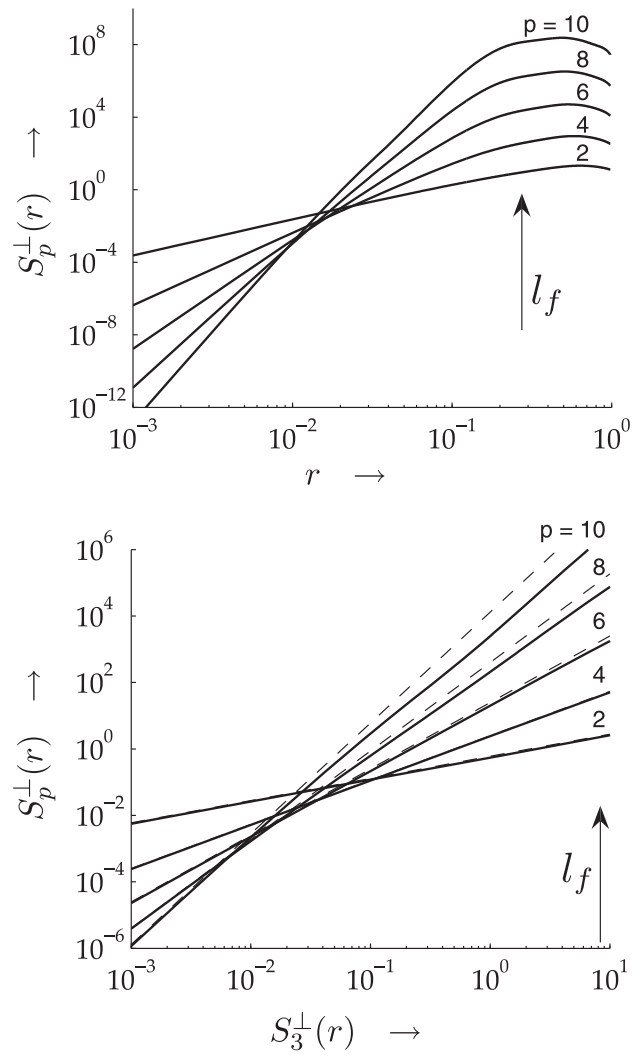

FIG. 8. Transverse structure functions $S_{p}^{\perp}$ of the velocity vs the separation $r$ (top panel) and versus the third-order structure function of the absolute value of the transverse velocity increments. The arrow denotes the forcing scale, and the dashed lines in the bottom panel correspond with a slope of $p / 3$. All results are obtained from simulations of forced 2D turbulence in a square bounded domain.

scaling exponent $\tilde{\zeta}_{p}$ for both the inverse energy cascade and direct enstrophy cascade range. It was observed that in the enstrophy cascade range the intermittency correction $\gamma_{p}$ was negligible, so that $\tilde{\zeta}_{p}=p / 3$. In the inverse energy cascade range a strong intermittency correction was found to be necessary. The magnitude of the intermittency correction in the inverse energy cascade is close to the corresponding value observed in 3D turbulence. Babiano et al. [35] developed an intermittency model for $\gamma_{p}$ based on the scaling behavior of local averages of the nonlinear transfer rate of the enstrophy. It correctly explains the observed absence of intermittency in the enstrophy cascade range and the presence of strong intermittency in the inverse energy cascade range.

Figure 8 (top panel) shows the structure functions with respect to the separation length $r$. In the viscous range the scaling exponent is equal to its upper bound, $\zeta_{p}=p$. For $r>$ $10^{-2}$ the scaling exponent is fractionally smaller and decreases strongly near the forcing scale. In Fig. 8 (bottom panel) the structure functions are plotted versus the third-order structure function. It can be seen that the scaling $\tilde{\zeta}_{p}=p / 3$ indicating self-similarity holds up to order $p=4$. Note that in the viscous range this relation is automatically satisfied due to the upper bound of the scaling exponent. For higher-order structure functions $(p \geqslant 6)$ deviations appear from the ESS scaling for $S_{3}^{\perp}(r) \gtrsim 10^{-2}$, which points toward some intermittency in the direct enstrophy cascade range. 


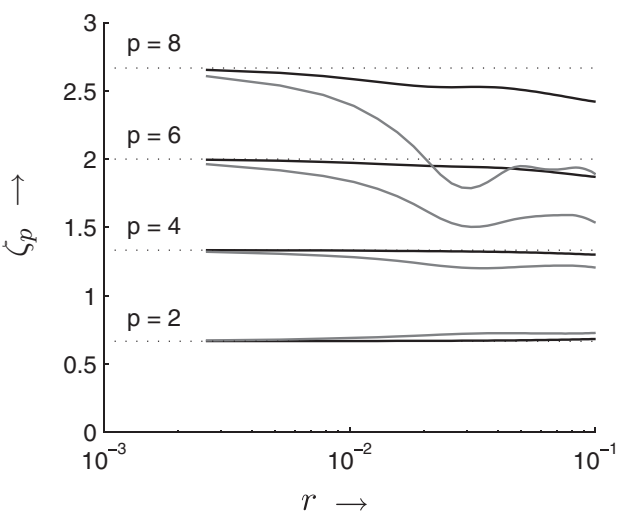

FIG. 9. ESS scaling exponent for $y=0$ (black) and $y=0.99$ (gray) for the structure functions of order $p=2,4,6$, and 8 . The dotted lines correspond with the $p / 3$. All results are obtained from simulations of forced $2 \mathrm{D}$ turbulence in the periodic channel domain.

Figure 9 gives the scaling exponent, determined using the ESS principle, found in the periodic channel. Let us first consider the structure functions obtained from the center line of the channel. As for the square domain deviations from ESS scaling appear for structure functions with $p \geqslant 6$. No deviations are observed for $p=2$ and 4 . The enstrophy cascade range is not long enough, however, to yield a plateau in the scaling exponent. Hence, there is no possibility to quantify the intermittency by calculating $\gamma_{p}$. Near the wall deviations are observed even for $p=2$. This indicates, as can be expected, that near the wall the turbulent flow is strongly intermittent.

\section{ENERGY AND ENSTROPHY FLUXES}

The second-order structure function of the vorticity, $S_{2, \omega}(r)=\left\langle(\delta \omega)^{2}\right\rangle$, is displayed in Fig. 10. This figure reveals that for the smallest scales $\left\langle(\delta \omega)^{2}\right\rangle \propto r^{1.5}$ and that it flattens for separations near the forcing scale $l_{f}$. The structure function $S_{2, \omega}(r)=\left\langle(\delta \omega)^{2}\right\rangle$ provides more information about the scaling exponents of the energy spectrum, since the slope of the second-order structure function of the vorticity is well separated from the upper bound. From Fig. 10 we can deduce that the corresponding energy spectrum has a slope of $k^{-3}$ near the forcing scale and becomes steeper for higher wave numbers, viz., $E(k) \propto k^{-4.5}$, which is significantly steeper than the inertial range prediction in the enstrophy cascade range by

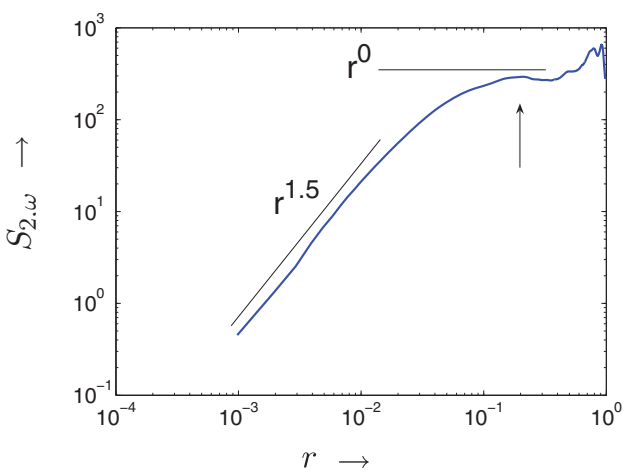

FIG. 10. (Color online) The second-order vorticity structure function $S_{2, \omega}$ in the interior of the square domain.
KBL. This might be due to the presence of a nonnegligible amount of viscous dissipation. The local dissipation scale is computed with $l_{d}=\left(v^{3} / \chi_{\text {loc }}\right)^{1 / 6}$, where the local enstrophy dissipation rate is defined as $\chi_{\text {loc }}=-v A^{-1} \int_{\mathcal{B}}|\nabla \omega|^{2} d A$, where $A$ denotes the area of the flow domain and $\mathcal{B}$ the measurement section in the interior of the domain. The ensemble averaged value for $l_{d}$ is approximately $5 \times 10^{-3}$, which is consistent with the position of the steep section of the vorticity structure function in Fig. 10. From these numbers it can be deduced that the enstrophy cascade extends over less than two decades between the forcing scale and the dissipation scale. Therefore, the role of viscous dissipation in the range between $l_{d}$ and $l_{f}$ cannot be excluded. On the other hand, in several studies it is anticipated that energy spectra steeper than $k^{-3}$ or second-order vorticity structure functions $S_{2, \omega} \propto r^{\alpha}$, with $\alpha>0$ result from the presence of coherent structures in the flow; see, e.g., Benzi et al. [36]. The high-resolution simulation of isolated vortex-merger events by Kevlahan and Farge [37], in which many intensive spiraling vorticity filaments are created, also show typically steeper energy spectra. Figure 2 clearly reveals the presence of coherent vortices and spiraling filamentary structures in the vorticity field. Therefore, the observed deviation from the KBL theory in the present simulations are not necessarily explained by viscous dissipation effects in the enstrophy cascade range. It might reflect the dominant role of coherent structures on the small-scale statistics.

Bernard [38] derived an analytical expression for a mixed velocity-vorticity structure function in the limit of $v \rightarrow 0$,

$$
\frac{\left\langle\delta u_{\|}(\delta \omega)^{2}\right\rangle}{r}=-2 \chi
$$

with $\chi$ denoting the ensemble-averaged enstrophy dissipation rate. It can be seen as an equivalent of the well-known 4/5 law of $3 D$ turbulence. The mixed third-order structure function, given by (15), can be interpreted as a measure of the nonlinear transfer rate of enstrophy. The role of the longitudinal velocity increments can be related to the alignment between the rate of strain tensor and the vorticity gradient vector, which describes the nonlinear amplification of the vorticity gradients. In the

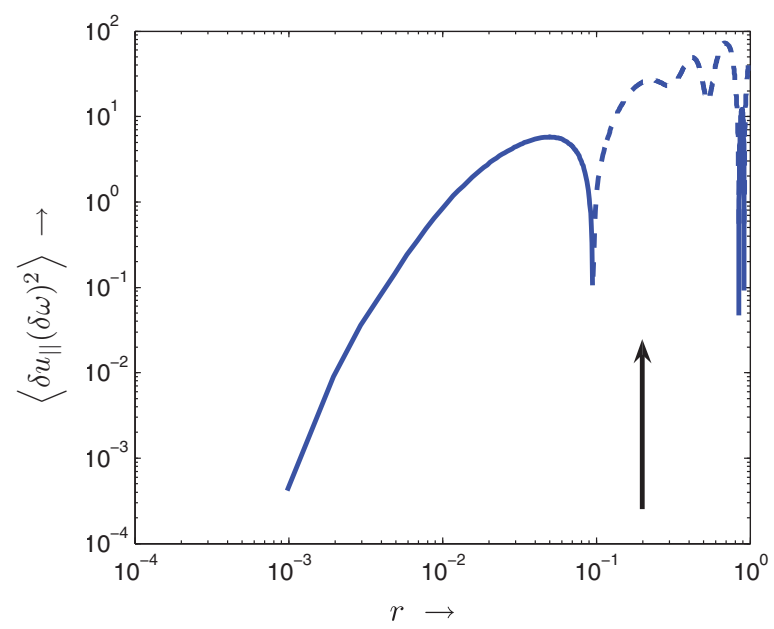

FIG. 11. (Color online) The mixed third-order structure function vs separation $r$. 


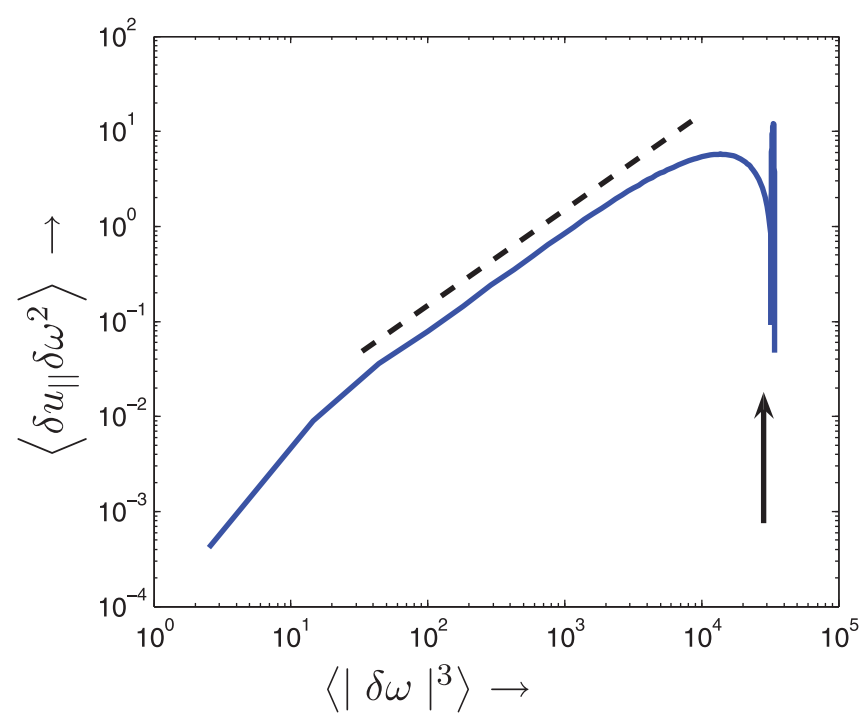

FIG. 12. (Color online) The mixed third-order structure function vs third-order structure function of the vorticity. Dashed line represents a slope of +1 .

derivation of relation (15) it is assumed that the flow is locally homogeneous and isotropic. In Fig. 4 it has been verified that these conditions can be assumed in the interior of the flow domain. Therefore, relation (15) can safely be applied to obtain an estimate for the enstrophy transfer rate. In Fig. 11 the mixed third-order structure function is shown with respect to the separation length. In the forcing range a sign-change of the mixed third-order structure function can be observed in Fig. 11. This can be related to the injection of vorticity by the external forcing. No other sign changes are observed in Fig. 11, which indicates that a direct enstrophy cascade is developed from the forcing range to the dissipation range.

The mixed third-order structure function does not clearly show the inviscid scaling predicted by Bernard [38], i.e., a scaling $\left\langle\delta u_{\|}(\delta \omega)^{2}\right\rangle \propto r$; see (15). To overcome the dissipation effect we use once again the concept of extended self-similarity discussed in Sec. VI. In this case the mixed third-order structure function is plotted versus the third-order structure function of the vorticity in Fig. 12. In the KBL framework for the enstrophy inertial range the vorticity structure functions are independent of the separation length. It can be deduced from Fig. 12 that the enstrophy flux demonstrates a scaling that is consistent with (15) in the limit of $v \rightarrow 0$. This signifies that the scaling for separations $r \lesssim l_{f}$ is consistent with the KBL picture of the direct cascade of enstrophy toward the smallest scale of motion. There is no sign that the vorticity injection from the sidewalls acts as a separate forcing length-scale for the internal flow.

Note that the analysis based on essentially isotropic relations for the mixed third-order structure function cannot be applied in the nonisotropic wall region.

\section{CONCLUSIONS}

Numerical simulations of forced flow in both a square domain and a periodic channel domain bounded by no-slip sidewalls reveal the existence of a statistically steady state that is maintained by a volumetric forcing and dissipation at the sidewalls. The structure of the turbulence under these conditions forms an important extension of the validation set for the KBL scaling theory since the effect of volumetric drag is absent in our simulations. It is found for the integral-scale Reynolds numbers considered in the present simulations that the flow is isotropic and locally homogeneous in the interior. These are important assumptions for the derivation of the KBL theory. However, in the enstrophy cascade range the turbulent flow is found to be intermittent. Also the scaling exponent of the energy spectrum, $E(k) \propto k^{-4.5}$, which was obtained from the vorticity structure function, is much steeper than the $k^{-3}$ spectrum from the KBL theory. From the simulations it is not yet clear whether this is solely due to viscous effects or that coherent vortices might play a role. The heavy tails in the pdf of vorticity increments are related to these coherent vortices, as was also observed in other studies on $2 \mathrm{D}$ turbulence in double periodic domains. To exclude viscous effects, simulations at higher Reynolds number are required. However, the restrictions on the grid resolution near the wall make these simulation presently hard to perform.

Near the wall, the detachment of vorticity boundary layers and the subsequent creation of small-scale vortices results in a high likelihood of large vorticity increments. It was observed that the flow is here strongly intermittent and anisotropic for all length scales. The small-scale vortices originating from the boundary layers also travel into the interior flow. They can be the cause of the observed weak intermittency in the interior for higher-order structure functions $(p \geqslant 6)$. These observations are in contrast with other studies of $2 \mathrm{D}$ turbulence in periodic domains (thus without no-slip sidewalls) where no significant intermittency in the enstrophy cascade range is found.

\section{ACKNOWLEDGMENTS}

The authors gratefully acknowledge financial support from the Dutch Foundation for Fundamental Research on Matter (FOM), which is financially supported by the Netherlands Organization for Scientific Research (NWO). WK and HJHC gratefully acknowledge financial support from the Netherlands Organisation for Scientific Research (NWO) and Technology Foundation (STW) under the Innovational Research Incentives Scheme grant ESF.6239. Part of this work was sponsored by the National Computing Facilities Foundation NCF for the use of supercomputer facilities, with financial support from NWO. The Fluid Dynamics Laboratory participates in the JM Burgerscentrum, research school for fluid dynamics, The Netherlands.
[1] U. Frisch, Turbulence: The Legacy of A. N. Kolmogorov (Cambridge University Press, Cambridge, 1995).

[2] L. F. Richardson, Weather Prediction by Numerical Process (Cambridge University Press, Cambridge, 1922).
[3] L. Onsager, Nuovo Cimento Suppl. 6, 279 (1949).

[4] R. Fjørtoft, Tellus 5, 225 (1953).

[5] R. H. Kraichnan, Phys. Fluids 10, 1417 (1967).

[6] R. H. Kraichnan, J. Fluid Mech. 47, 525 (1971). 
[7] G. K. Batchelor, Phys. Fluids Suppl. II 12, 233 (1969).

[8] C. E. Leith, Phys. Fluids 11, 671 (1968).

[9] D. Lilly, Phys. Fluids Suppl. II 12, 240 (1969).

[10] G. Boffetta, J. Fluid Mech. 589, 253 (2007).

[11] H. J. H. Clercx and G. J. F. van Heijst, Appl. Mech. Rev. 62 020802 (2009).

[12] L. M. Smith and V. Yakhot, Phys. Rev. Lett. 71, 352 (1993).

[13] L. M. Smith and V. Yakhot, J. Fluid Mech. 274, 115 (1994).

[14] M. Hossain, W. H. Matthaeus, and D. Montgomery, J. Plasma Phys. 30, 479 (1983).

[15] H. J. H. Clercx and G. J. F. van Heijst, Phys. Rev. Lett. 85, 306 (2000).

[16] M. G. Wells, H. J. H. Clercx, and G. J. F. van Heijst, J. Fluid Mech. 573, 339 (2007).

[17] W. Kramer, H. J. H. Clercx, and G. J. F. van Heijst, Phys. Fluids 20, 056602 (2008).

[18] W. Kramer, Ph.D. thesis, Eindhoven University of Technology, The Netherlands (2007).

[19] G. H. Keetels, U. D’Ortona, W. Kramer, H. J. H. Clercx, K. Schneider, and G. J. F. van Heijst, J. Comput. Phys. 227, 919 (2007).

[20] O. Daube, J. Comput. Phys. 103, 402 (1992).

[21] W. Kress and P. Lötstedt, Comput. Methods Appl. Mech. Eng. 195, 4433 (2006).

[22] H. J. H. Clercx, J. Comput. Phys. 137, 186 (1997).

[23] G. Carbou and P. Fabrie, Adv. Differ. Equ. 8, 1453 (2003).
[24] G. H. Keetels, H. J. H. Clercx, and G. J. F. van Heijst, Eur. J. Mech. B/Fluids 29, 1 (2010).

[25] W. J. T. Bos, S. Neffaa, and K. Schneider, Phys. Plasmas 17, 092302 (2010).

[26] K. Schneider and M. Farge, Phys. Rev. Lett. 95, 244502 (2005).

[27] B. Kadoch, W. J. T. Bos, and K. Schneider, Phys. Rev. Lett. 100, 184503 (2008).

[28] W. J. T. Bos, S. Neffaa, and K. Schneider, Phys. Rev. Lett. 101, 235003 (2008).

[29] G. H. Keetels, H. J. H. Clercx, and G. J. F. van Heijst, Physica D 238, 1129 (2009).

[30] G. H. Keetels, H. J. H. Clercx, and G. J. F. van Heijst, Phys. Rev. E 78, 036301 (2008).

[31] G. H. Keetels, Ph.D. thesis, Eindhoven University of Technology, The Netherlands (2008).

[32] A. Babiano, C. Basdevant, and R. Sadourny, J. Atmos. Sci. 42, 941 (1985).

[33] G. Boffetta, A. Celani, S. Musacchio, and M. Vergassola, Phys. Rev. E 66, 026304 (2002).

[34] R. Benzi, S. Ciliberto, R. Tripiccione, C. Baudet, F. Massaioli, and S. Succi, Phys. Rev. E 48, 29 (1993).

[35] A. Babiano, B. Dubrulle, and P. Frick, Phys. Rev. E 52, 3719 (1995).

[36] R. Benzi, G. Paladin, and A. Vulpiani, Phys. Rev. A 42, 3654 (1990).

[37] N. Kevlahan and M. Farge, J. Fluid Mech. 346, 49 (1997).

[38] D. Bernard, Phys. Rev. E 60, 6184 (1999). 